\title{
A Case of Phaeochromocytoma presenting as incidentloma
}

S Ahmed, B K El-Mahmoudi

Tameside Hospital NHS Foundation Trust, Ashton-Under-Lyne, Manchester, OL6 9RW, UK- The University of Manchester

\section{Case report}

83 year old gentleman admitted with nausea and feeling generally unwell. Past medical history of hypertension, duodenal ulcer, previous gastric surgery, polymylagia rheumatica and type 2 diabetes mellitus. Chest XR showed right basal pneumonia which was treated with antibiotics. Patient admitted to significant weight loss therefore he had thoracic-abdominal scan performed which showed $4 \mathrm{~cm}$ by $4 \mathrm{~cm}$ solid cystic lesion in the left adrenal gland possibly malignant. Subsequently magnetic resonance imaging scan revealed left adrenal heterogeneous lesion which could be either primary or metastatic. $24 \mathrm{hr}$ urinary metadrenaline $7.2 \mathrm{umol} / 24 \mathrm{hrs}$, normetadrenaline $4.1 \mathrm{umol} / 24 \mathrm{hrs}$. These findings were consistent with diagnosis of phaeochromocytoma. He had experienced hypotensive episodes associated with dizziness these were treated with intravenous fluids to expand his intravascular volume. He did not tolerate phenoxybenzamine because of dizziness and low blood pressure. He was assessed for surgical treatment to his phaeochromocytoma but in view of his multiple co-morbidity he declined surgery therefore he was managed conservatively. For nearly 2 years he remains under outpatient follow-up and his repeated imaging showed slight enlargement of his original lesion but no new lesion

\section{Learning Points}

-The classical triad of paroxysmal headache, palpitation and sweating may not be present in elderly patients.

-Phaeochromocytoma can be discovered incidentally on imaging done for unrelated symptoms.

-24 hour urinary metanephrines has high sensitivity and is now increasingly the prefered test. The tumour can be localised with MRI scan or iodine-131 MIBG test (metaiodobenzylguanidine).

-Management of Phaeochromocytoma in the elderly with complex co-morbidity can be challenging.

\section{Discussion}

Combination of headache , sweating and palpitation has a specificity of more than $90 \%$ for diagnosis of phaeochromocytoma Hypertension in some form is present in $80-90 \%$, palpitations in $60 \%$.

a multiple endocrine neoplasia (MEN-type2A).

$-10 \%$ of phaeochromocytomas occur outside the adrenals, mostly in the abdomen

-Approximately $10 \%$ tend to be malignant and can metastesise to liver and bone

.

Patients not fit to undergo surgery are managed conservatively.

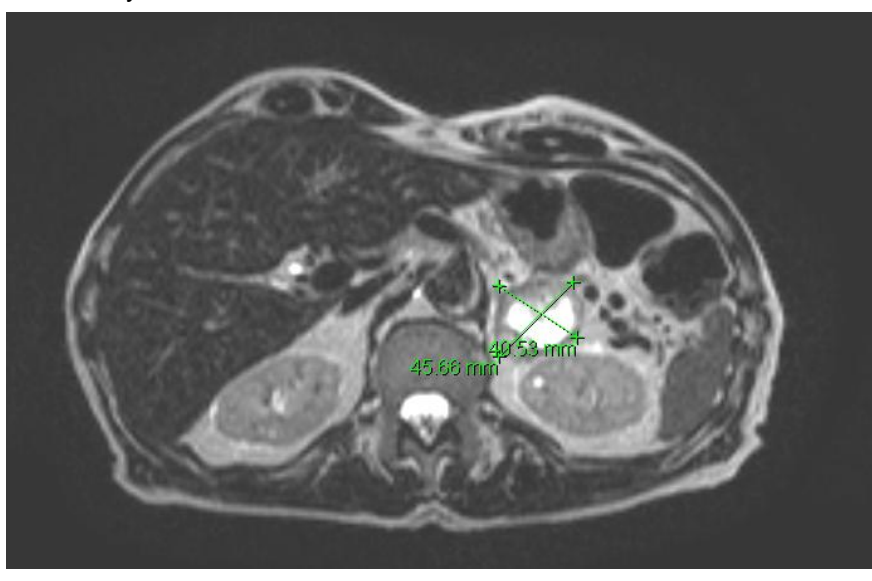

\section{References}

1 phaeochromocytoma and paraganglioma..Medicine,,volume 37 issue 8.Pages 420-421 2Adrenal incidentalomas:The rule of four. Clinical Medicine, volume 8, number 2, 2008 3Phaeochromocytoma: Journal of Royal college of Physicians of London,. Vol 32 ,No.2,pages 106-107, April 1998

4 Easily missed. Phaeochromocytoma. BMJ 2012;344 